REVISTA DE DERECHO UNED, NÚM. 17, 2015

\title{
EL PREMIO NOBEL DE LA PAZ 1901-1914. VOLUNTAD O INTERÉS
}

\author{
THE PEACE NOBEL PRIZE 1901-1914. WILL OR INTEREST
}

\section{EugENIO HERNÁNDEZ GarCÍA}

Doctorando de la Facultad de Derecho de la UNED

Resumen: Los primeros premios Nobel de la Paz, (al igual que los otros premios Nobel), se concedieron en diciembre de 1901, entre esa fecha y el inicio de la I Guerra Mundial en 1914, el premio se consolida, igualmente se sientan las bases de lo que será en el futuro. Las líneas que siguen tratan de demostrar cómo, a pesar de tener su origen y fundamento en el testamento ológrafo de Alfredo Nobel, datado sólo seis años antes, y donde se establecían los criterios a considerar, lo cierto es que la Comisión encargada de su concesión, como cuerpo de origen político, transformará el siempre difícil idealismo de la voluntad, en un más práctico realismo político, haciendo una redefinición de los méritos y voluntad de su autor, de quien quedará, eso sí, la idea, el nombre y el dinero. La actividad privada particular cederá ante la política, y la fraternidad entre las naciones, la abolición o reducción de los ejércitos permanentes y la celebración y promoción de congresos de paz, se transformaran en derecho internacional y arbitraje.

Palabras clave: Alfredo Nobel. Premios Nobel de la Paz. Pacifismo.

Abstract: The first Peace Nobel Prizes (as well as the rest of Nobel Prizes), were given in December 1901. Between that date and the beginning of the First World War in 1914 the Prize consolidates together with the basis of what it will become in the future. The followed lines try to show how, despite having its origin and grounds in Alfred Nobel's holographic will, dated only six years before, and when criteria to be considered were established, the truth is that the Commis- 
sion in charge of its award, as a body with political origin, will turn the always difficult will idealism into a more practical political realism, making a new definition of its author's merit and choice, whose idea, name and money will remain. The private activity will fall under politics, and brotherhood among nations, the abolition or reduction of permanents armies, the celebration and promotion of Peace Congresses will turn into International Law and Arbitration.

Key words: Alfred Nobel. Nobel Peace Price. Pacifism.

Recepción original: 18/09/2015

Aceptación original: 20/10/2015

Sumario: I. Introducción. II. El testamento, nominadores, nominaciones, Comité. III. Los premiados IV. Conclusiones.

\section{INTRODUCCIÓN}

En 1901, se concedieron los primeros premios Nobel, entre ellos el de la Paz ${ }^{1}$ quizá el más universal y en ocasiones polémico. El origen de todos ellos, se encuentra en una disposición testamentaría de Alfred Nobel, y como tal, su ejecución (lo más fiel dentro de lo posible) es cometido de aquellos que previa designación por el testador aceptaron el mandato, que en este caso corresponde a una comisión (Comité) elegida por el Parlamento noruego. Se pretende a partir de este momento, en base a la composición del Comité y la condición de los premiados, determinar en qué medida la citada comisión en una fase inicial, la comprendida entre 1901 y la I Guerra Mundial, se ha ceñido al mandato recibido, y que línea ha seguido en la concesión de los premios. En otras palabras si en su concesión se ha atendido a la voluntad de Nobel o por el contrario el Comité ha fijado sus propios criterios.

\section{EL TESTAMENTO, NOMINADORES, NOMINACIONES Y COMITÉ}

Un premio Nobel se apoya sobre tres pilares; en primer lugar el testamento, que determina los requisitos para su concesión, un se-

${ }^{1}$ Nobel no le dio nombre, se limitó a afirmar que se concedieran a «la persona que ha hecho más o mejor aportación para la fraternidad de las naciones y la abolición o reducción de los ejércitos permanentes así como la celebración y promoción de congresos por la paz», aunque cuando determina a quien corresponde otorgarlo le calificará como «El defensor de la paz». Sigo la interpretación que del mismo se hace en «La voluntad de Alfred Nobel» de Fredrik S. Heffermehl, Ed. Icaria, Barcelona, 2013. 
gundo el Comité, o comisión, que debe decidir a quien se concede el premio y por último la nominación, de forma que quién no ha sido nominado previamente y en los plazos establecidos, -como máximo el día uno de febrero-, no puede ser premiado, lo que impone una referencia aunque sea breve sobre quienes tienen la facultad de nominar, advirtiendo previamente que en todo caso las nominaciones son un límite relativo para el Comité, pues hasta la primera reunión de este organismo tras la finalización del periodo de nominaciones, cualquiera de sus miembros puede nominar; una vez superado ese plazo la falta de nominación impide el premio para ese año.

Los antecedentes necesarios se sitúan en 1895, cuando Alfred Nobel redacta y firma la que será su última y definitiva disposición testamentaría ${ }^{2}$, en ella determina el destino de su fortuna ${ }^{3}$ y establece los premios $^{4}$ que llevarán su nombre, junto con los criterios de concesión que pueden resumirse del siguiente modo: Con validez para todos ellos, en cuanto a su duración, aunque nada se señala, es evidente que tendrán carácter indefinido o tal vez hubiera que decir permanente, por cuanto el importe de los premios no se satisface con el capital o fondo constituido tras la enajenación de sus bienes, sino con sus «intereses», lo que a priori pone de manifiesto su intención de permanencia.

En cuanto a los requisitos, válidos también para todos los premios, indica que se reparta entre «aquellos que durante el año transcurrido hayan realizado el mayor beneficio a la humanidad» aunque no se da apenas importancia a este párrafo, que sistemáticamente es omitido en las presentaciones y discursos, especialmente en el de la Paz; a mi juicio la tiene para una correcta interpretación de su voluntad, porque hay que entender que está pensando en premiar hechos o descubrimientos acaecidos entre un premio y otro, o en todo caso recientes, con ello lo que se persigue es que la actividad pacifista sea constante y actual; quizá tomando como referencia su discurrir investigador, evitando que recaiga sobre realizaciones ya superadas, y a continuación, ya concretando en el conocido como de la paz, fija que se entregara «... a la persona que ha hecho más o mejor aportación para realizar la fraternidad de las naciones y la abolición o reducción de los ejércitos permanentes así como la celebración y promoción de los congresos de

${ }^{2}$ El testamento fue firmado en París el 27 de noviembre de 1895.

${ }^{3}$ Conforme al inventario efectuado presentado ante el Tribunal provincial de Karlskoga, los bienes de Nobel ascendían a la cantidad de 31.587.202,28 coronas. SOHLMAN, Ragnar y otro. Los premios Nobel y su fundador. Versión en castellano, Madrid, 1959, pág. 101.

${ }^{4}$ Física, Química, Fisiología o Medicina, Literatura y «el del defensor de la paz». 
paz». De la literalidad del mismo deja claro que se ha de tratar de una persona física, y afinando más a sólo a una por año, en todo caso no cabría el premio para una asociación u organización. Sin embargo, el Reglamento para la adjudicación del Premio Nobel de la Paz ya desde el principio reconoce que puede «adjudicarse a instituciones y asociaciones $»^{5}$. Por último de su concesión se encargará «una comisión de cinco personas elegidas por el Parlamento noruego».

Cuando superadas no pocas dificultades ${ }^{6}$, se constituya esta comisión (denominada Comité), no será otra cosa que un reflejo del panorama político y social dominante en Noruega a principios de $\operatorname{siglo}^{7}$, Parlamento, gobierno y universidad-; a partir de $1901^{8}$ quedará integrada por: Jørgen G. LØVLAND ${ }^{9}$ destacado líder del partido Liberal, parlamentario, procedente de la docencia y del periodismo, que hará compatibles sus puestos gubernamentales con el Comité. Ministro de Obras Públicas, ocupará el cargo de Ministro de Asuntos Exteriores del país tras la independencia (1905-1908), y Primer Ministro al final de este periodo. En el Comité, ejercerá como presidente durante todos estos años hasta su fallecimiento en 1922. John LUND: Liberal. Parlamentario, presidente del Lating, ${ }^{10}$ y delegado de Noruega en diversas conferencias de paz, formará parte del Comité hasta 1912, en 1913 entrará Cornelius Bernhard Hansen también del Partido Liberal, profesor, editor, propietario vinculado al sector naviero, jugará un papel relevante en la Sociedad Noruega de la Paz; Bjørnson BJØRNSTJERNE: Liberal, periodista, poeta, político, recibió el Nobel de Literatura en 1903. Por su prestigio como escritor es sin duda el más conocido internacionalmente, y aunque pasa temporadas fuera

${ }^{5}$ SOHLMAN, Ragnar y otros. Los premios Nobel y su fundador, Ed. Aguilar, Madrid, 1959, pág. 984.

${ }^{6}$ Ídem, págs. 33-120.

${ }^{7}$ A la altura de 1900, Noruega tenía unos 2.230.000 habitantes muy diseminados, con un escaso desarrollo económico, pobre agricultura y volcada al mar, lo que da una idea de la concentración en términos de poder y cultura que aglutinan el parlamento y la universidad.

${ }^{8}$ La primera Comisión se constituye en 1897, formada por Bernhard GETZ como presidente, Jørgen LØVLAND vicepresidente, y los vocales John LUND, Johannes STEEN, y Bjørnstjerne BJØRNSON. A la muerte de GETZ en 1901, LØVLAND pasa ocupar la presidencia y entra como vocal Hans Jacob HORST.

${ }^{9}$ Las datos biográficos sobre los miembros del Comité proceden de las siguientes fuentes: Página del Premio nobelprize.org.; LIBÆK Ivar. The Nobel Peace Prize: Some aspects of the decision-making process, 1901-17. The Norwegian Nobel Institute Series, Vol 1, n. ${ }^{\circ}$ 2, Oslo, 2000. y REINAR, Mathias Brynildsen, «Lille land - hva da? Nobelinstituttet og begynnelsen på den norske fredstradisjonen.» Institutt for sosiologi og statsvitenskap, NTNU, 2011.

${ }^{10}$ Aunque el Parlamento noruego es unicameral está dividido en dos secciones el «Lating» especie de «Cámara alta» y el «Odelsting» o «Cámara baja». 
de Noruega, se mantendrá atento a las discusiones del Comité. Ocupará puesto como miembro hasta 1906. Johannes V. C. STEEN: Liberal, profesor, parlamentario, Presidente de Parlamento, Ministro de Finanzas, Primer Ministro. Hans Jacob HORST: Igualmente liberal, profesor, parlamentario, Presidente de ambas cámaras, Presidente de la Asociación de la Paz del Parlamento, miembro de la Corte Internacional de Arbitraje de la Haya y vicepresidente de la Sociedad Noruega de la Paz. Carl Berner: Liberal, Presidente del Parlamento, en 1904, sustituirá provisionalmente a Bjørnson y a partir de 1906 ocupará puesto en el Comité. Por último Georg Francis HAGERUP, el primer conservador en entrar el Comité como miembro efectivo, para la elección de los premiados, por cuanto Getz, como se ha dicho falleció prematuramente, se integra en 1907, permaneciendo hasta 1921. Doctor en Derecho y profesor en la Universidad de Oslo, interesado en el derecho internacional, fue Ministro de Justicia y Primer Ministro, encabezará en 1907 la delegación noruega en la Conferencia de Paz de la Haya, también participó en diversas conferencias sobre derecho del mar, miembro del Instituto de Derecho Internacional y a partir de 1912 presidente de este organismo ${ }^{11}$, representó a Noruega en el Tribunal de Arbitraje de la Haya. También a partir de 1901se incorpora la figura del Asesor, puesto que inicialmente ocuparán el historiador y futuro miembro del Comité Halvdan Koht, el relevante jurista Nikolaus, que aparte de sustituir a Steen en 1904, figura clave en el proceso de independencia del país, y el no menos prestigioso economista, historiador, profesor de la Universidad de Cristianía, y por breve tiempo ministro Ebbe HERTZBERG, que pronto cesará. Cuando esto ocurra y Carl Berner pase a ocupar puesto como vocal del Comité, serán nombrados nuevos asesores, el experto en derecho internacional Mikael H. LIE y el economista, periodista y político Karl Vilhelm HAMMER, a los que se unirá en 1907 Christian L. LANG al dejar la secretaria.

La extensa referencia anterior sólo tiene como objetivo poner de manifiesto como con independencia de su relación con el pacifismo, todos los miembros del Comité, secretarios y asesores, se encuentran estrechamente vinculados a la clase política ${ }^{12}$, casi todos del partido

${ }^{11}$ MLA style. «Bio-George Francis Hagerup». Nobelprize.org. Nobel Media AB 2014. Web. 23 Aug 2015. http://www.nobelprize.org/nobel_prizes/themes/peace/ committee/nnclist/bios/hagerup.html.

${ }^{12}$ MLA style. "The Norwegian Nobel Committee». Nobelprize.org. Nobel Media AB 2014. Web. 31 Jul 2015. http://www.nobelprize.org/nobel_prizes/themes/peace/ committee/index.html. 
liberal ${ }^{13}$, y algunos muy relacionados simultáneamente con la docencia, el periodismo e incluso la empresa, no conviene olvidar que Noruega tendrá una flota mercante de primer orden, ello unido a su participación en conferencias de paz de la época, incluidas las de la Haya, el fuerte peso de los partidarios del derecho internacional y su vinculación con organizaciones pacifistas, especialmente la Unión Inter-parlamentaria y la Oficina Internacional de la Paz, al extremo que John Lund y Hans Horst formaran parte del Consejo de la primera donde compartirán puesto con Passy, Gobat y Cremer ${ }^{14}$.

Asimismo el Comité contará con un Secretario, cargo que inicialmente recaerá en Christian L. Lange, que hasta 1904 será quien asuma la tarea de realizar los informes en que supuestamente se basa la elección de los premiados, cuenta con amplia formación académica y después asumirá la Secretaria General de la Unión Inter-parlamentaria. Como secretario de Comité ocupará la dirección del Instituto Nobel de Noruega, fundado el 1 de febrero de $1904^{15}$ A partir de 1910 y durante los treinta y cinco años siguientes el puesto será ocupado por el influyente profesor e historiador Ragnvald Moe.

El tercer pilar es el correspondiente a los nominadores, con dos cuestiones a considerar, por una parte quien o quienes tienen la facultad nominar y por otra hacer una aproximación a los primeros nominados.

El punto de partida para acceder al premio pasa por conseguir una nominación. Aquí aparece el primer filtro, la capacidad de nominar está limitada a: Los miembros de asambleas nacionales y de los gobiernos de los estados; miembros de los tribunales internacionales; rectores de universidades, profesores de ciencias sociales, historia, filosofía, derecho y teología, directores de institutos de investigación de la paz y de política exterior; personas que han recibido el Premio Nobel de la Paz; miembros del Consejo de organizaciones que hayan sido premiadas con el Premio Nobel de la

${ }^{13} \mathrm{El}$ partido liberal, aunque apoye una legislación social, y pueda situarse en la izquierda parlamentaria representa una posición política claramente conservadora partidario de la libertad económica, favorable a la empresa capitalista, y lógicamente opuesto a las ideas socialistas. El sufragio masculino estuvo limitado hasta 1898 y para encontrar socialistas en el parlamento noruego habrá que esperar a 1903 con una mínima representación de cuatro miembros. Agrupados como Partido Laborista en 1906 pasó a 10 escaños, tres años después a 11 y en 1912 a 23 . No será hasta 1927 cuando se convierta en la primera fuerza política del país.

14 «El Liberal» 19-09-1908.

15 http://nobelpeaceprize.org/en_GB/institute/. 
Paz; miembros activos y retirados del Comité Nobel, y por último ex asesores del Comité Nobel ${ }^{16}$.

La relación anterior muestra el carácter cerrado de los nominadores, los cargos que ocupan evidencian que se trata de la elite social, política y cultural, extremo que se multiplica si tenemos en cuenta que el derecho de voto estaba limitado bien a un sufragio censitario o en el mejor de los casos a un sufragio universal masculino, y la mujer carecía del derecho de voto. La composición parlamentaria distaba de reflejar una pluralidad social, los partidos socialistas y comunistas no existían o su presencia era testimonial, y el mundo universitario era igualmente restringido en unos tiempos en que apenas se aspiraba a la alfabetización total.

Con estos nominadores el siguiente paso es repasar brevemente las nominaciones efectuadas el primer año; esto es las presentadas para el premio de 1901, y de las que al menos sobre el papel cabría esperar una gran dispersión. Tres cuestiones saltan a la vista, por un lado que el premio despierta gran interés desde el principio, puesto que contabiliza 137 nominaciones ${ }^{17}$, si bien la concentración es grande pues los candidatos se reducen a 32, un segundo aspecto y más llamativo es que entre los nominados de este año aparecen aparte lógicamente de los galardonados los de los cuatro siguientes, y algunos más que serán premiados en posteriores ocasiones. La correlación entre nominados y nominadores como clase social o como colectivo es casi total, (políticos y juristas, muchos de ellos con vínculos políticos son holgada mayoría) y lo mismo ocurre en consecuencia con los premios, el primer puesto corresponde a Passy, que recibe 41 nominaciones, bastante dispersas, de profesores de la Universidad de Zagreb, miembros del parlamento húngaro, del parlamento francés, del parlamento sueco, austriaco y múltiples individuales incluidas las de dos futuros Nobel, Bajer y Suttner ${ }^{18}$, por su parte Dunant, sólo recibirá 10, pero algunas de peso como la «92 miembros del parlamento de Suecia», de miembros del parlamento de Württemberg a las que se unirá la de Bernhard Getz, primer presidente del Comité del premio Nobel y que falleció pocos meses antes de la concesión del premio. Los que recibirán los siguientes premios son Ducommun y Gobat en 1901 ya recibirán 8 y 1 nominaciones;

${ }^{16}$ MLA style. "Nomination and Selection of Peace Prize Laureates». Nobelprize. org. Nobel Media AB 2014. Web. 25 Aug 2015. http://www.nobelprize.org/nomination/ peace/index.html.

${ }^{17}$ MLA style. «Nomination Database». Nobelprize.org. Nobel Media AB 2014. Web. $30 \mathrm{Jul}$ 2015. http://www.nobelprize.org/nomination/archive/list.php.

${ }_{18}$ MLA style. «Nomination Database». Nobelprize.org. Nobel Media AB 2014. Web. $30 \mathrm{Jul}$ 2015. http://www.nobelprize.org/nomination/archive/show_people.php?id=7015.

(C) UNED. Revista de Derecho UNED, núm. 17, 2015 
Cremer premiado en 1903 tendrá 15 nominaciones, el Instituto Internacional de Derecho premio en 1904 contabilizará 3 nominaciones, por su parte Suttner que recibirá el premio en 1905 tendrá 4; y aún aparecen nominados otros que serán premiados antes de la I Guerra Mundial, como la Oficina Internacional de la Paz en 1910 con 14, o Bajer con 4. Hay por último una nominación que pasa casi desapercibida la correspondiente a la Sociedad Inter-regional por la Paz. Unión Lombarda, que aunque como tal asociación no recibirá el premio, sí lo hará en 1907 su fundador, Ernesto Teodoro Moneta. Merece mencionarse aparte de la nominación de Dunant la de la Cruz Roja, que tendrá que esperar a 1917 para recibir su primer premio. Con esta relación se pone de manifiesto, de forma palmaria en qué medida una mayoría relevante de los nominadores y el Comité sintonizan.

En los años inmediatos, tan pronto como se vea la línea seguida, irán disminuyendo las nominaciones en 1902 serán 105 para caer en 1903 a 65, si bien no tanto el número de propuestos, se irán cayendo algunos que es evidente que no van a recibir el premio, como el Zar Nicolas II, que inspiró la primera Conferencia de Paz de la Haya de 1899, pero de cuya dictadura absolutista era enemigo hasta el mismo Nobel ${ }^{19}$, o Tolstoi cuyo radicalismo político no podía gozar de las simpatías de la élites conservadoras. Nominado en 1901 y 1902 por E. H. Hiltz, profesor de la Universidad de Berna en Suiza, no tendrá opciones. Poco después se incorporan rápidamente a las nominaciones figuras que encajan dentro de los criterios que invariablemente sigue el Comité, como Klas Pontus Anorldson o Moneta.

Dos cosas quedan ya claras, la uniformidad de los nominadores y que no tienen en cuenta actuaciones recientes, son figuras y organizaciones ya existentes, incluso algunos habían nacido antes que el propio Nobel, irán recibiendo el premio unos detrás de otros y sólo la aparición de nuevos e insospechados acontecimientos o su muerte impedirá que el reparto se concentre aún más. Con el testamento como base, y las nominaciones que se habían realizado, queda por ver qué criterios van a guiar el hacer del Comité. esto es en que medida asume como suyas estas nominaciones.

\section{LOS PREMIADOS}

Cuando Nobel firma el testamento en 1895 e incluso cuando fallece al año siguiente, y hasta 1905, Noruega se encontraba bajo dominio político de Suecia desde hacía casi un siglo, si bien a pesar ello

${ }^{19}$ SOHLMAN, Ragnar y otros. Los premios Nobel ..., cit., pág. 25. 
disponía de gran autonomía de gobierno, contaba con parlamento propio y salvo la representación exterior y la jefatura del gobierno en los demás aspectos era prácticamente un país soberano. Su sistema parlamentario, aunque la mujer no tenía derecho de voto, podía considerase en su época como democrático y si carecía de una política exterior propia había desarrollado una cultura amplia de relación con el movimiento pacifista de influencia y desarrollo creciente en estos años. Así pues distinguimos conforme a esto entre los premios concedidos antes y después de la independencia.

En 1901, se hacen públicos los nombres de los primeros premiados; se anuncian el 10 de diciembre, si bien a diferencia de lo que ocurre actualmente, el nombre de los premiados se desconocía hasta ese momento, por lo que estos no están presentes, además hay que contar con las dificultades en los desplazamientos que existían en la época, y ello unido a la elevada edad de algunos de los premiados, hace que cuando estos acudan a Cristianía, para recibir el premio hayan pasado meses o años desde la concesión. De este modo en estos primeros momentos, en esa fecha se limitaba a designar a los premiados, tampoco se dispondrá de mucha información sobre las causas, y habrá que esperar en algunos casos a la recepción del premio para tener mejores noticias sobre la motivación y aun así no siempre de forma directa ${ }^{20}$.

Lo que sí se deja entrever desde el principio, es que el premio juega un papel importante en la política de Noruega, y como tal será usado. Ya en 1901, el premio es otorgado en una reunión del Parlamento noruego en la que el presidente del Parlamento, Carl Christian Berner, aprovecha el acto para hacer una llamada a la independencia de Noruega, «El pueblo noruego siempre ha exigido que se respete su independencia» después el presidente del Comité hará público el nombre de los premiados ${ }^{21}$. Este año los beneficiados son Frederik Passy y Henry Durant; las vicisitudes que llevaron a la concesión del premio a Dunant, que a priori no contaba con posibilidades por cuanto se consideraba que su actividad no respondía a la voluntad de Nobel, fue el resultado de una exitosa campaña que ha llamado la atención de algunos especialistas ${ }^{22}$. Será sin embargo con el tiempo uno de los menos discutidos su carácter universal y el relevante papel que

${ }^{20}$ Esta información esta recopilada para toda la historia del premio por HABERMAN, Frederick W. Peace. (6 Vol.), Edit. World Scientific., 1999.

${ }^{21}$ MLA style. «The Nobel Peace Prize 1901». Nobelprize.org. Nobel Media AB 2014. Web. 21 Aug 2015. http://www.nobelprize.org/nobel_prizes/peace/laureates/1901/address.htm.

${ }^{22}$ Véase LIBÆK Ivar. The Nobel Peace Prize: Some aspects of the decission-making process, 1901-17. The Norwegian Nobel Institute Series, Vol 1 N. ${ }^{\circ}$ 2, Oslo 2000.; DURAN, André. El primer Premio Nobel de la paz (1901) - Las candidaturas de Henry 
en la historia jugará la Cruz Roja, a la larga dará renombre al premio, dentro y fuera del mundo del pacifismo.

Cuando Passy ${ }^{23}$ recibe el premio es casi un octogenario, polifacético con formación en filosofía, derecho y economía, que ha ejercido como diputado, y cuando no sea reelegido en 1889, se dedicara a la docencia, publicará algunas obras sobre economía y pacifismo, y sobre todo será conocido por el papel desempeñado en las dos principales organizaciones pacifistas del momento la Unión Inter-parlamentaria y la Oficina Internacional de la Paz, asimismo en su haber se encuentra la fundación de la Liga Internacional Permanente de la Paz, que pasaría a ser la Sociedad Francesa de Amigos de la Paz y por último se transformaría en la Sociedad Francesa para el Arbitraje entre las Naciones. Así encontramos por un lado el recurso al premio compartido cuando se trata de buscar acuerdos dentro de las diversas posturas en el Comité o sea preciso dar cabida a premiados meritorios o de gran repercusión mediática, que pueden mejorar la imagen internacional de Premio, además se incumple, ya desde el principio la condición de premiar un «haber hecho» más y mejor durante «el año transcurrido», se dirige a una personalidad con una dilatada trayectoria en el mundo de las organizaciones pacifistas, muy reconocido, que transmite prestigio en ese círculo, además el valor que se considera como relevante es el recurso al arbitraje como medio de resolución de conflictos.

El Comité no evolucionará y durante los próximos años, los premios no son más que un calco del concedido a Passy. En 1902, se reparte entre otros dos históricos de este movimiento Élie Ducommun y Albert Gobat, que por cierto, han sido nominados también por los miembros del Comité John Lund y Hans J. Horst, miembros de la Inter-parlamentaria y por el grupo Inter-parlamentario sueco.

La presentación de aquél tendrá lugar el 16 de mayo de 1904, en ella por el presidente del Comité, Jørgen G. Løvland se reconocen las que acertadamente denomina dos ramas del pacifismo burgués, la

Dunant, de Gustave Moynier y del Comité Internacional de la Cruz Roja.30-06-2001 Revista Internacional de la Cruz Roja.

${ }^{23}$ Los datos biográficos de los galardonados proceden aparte de la propia página del premio www.nobelprize.com de las siguientes obras SALVAGGIO, Santos. Diccionario biográfico de los Premios Nobel. Ed. Claridad, S. A, Buenos Aires, 1958; SALVAGIO, Santos. Los premios Nobel: la fundación y biografías de los galardonados, Edt. Ramon Sopena, Barcelona, 1981; Biographical Dictionary of Internationalist, Edited by Warren F. Kuehl, Connecticut, United States of América 1983.; Biographical Dictionary of Modern Peace Leader. Editor in chief Harold Josephson Connecticut. United States of América 1985; MADRILEJOS, Mateo, en Los premios Nobel... Dir. Juan M Prado, Editorial ORBIS, Barcelona, 1983. 
parlamentaria y la popular ${ }^{24}$, esta como aglutinante de todas la sociedades pacifistas del mundo ${ }^{25}$. Ducommun representa esta segunda por su papel en la fundación de la Liga Internacional de la Paz y la Libertad pero sobre todo por su trabajo como secretario de la Oficina Internacional de la Paz. Pero como el Comité ha asumido la necesidad de incorporar como valor fundamental el arbitraje y el derecho internacional, no podía premiarse solamente a Ducommun, era necesario incluir una figura más relevante desde el punto de vista jurídico y, por supuesto, dejar claro el apoyo del Comité a la Unión Inter-parlamentaria, así se justifica el premio para el Secretario General Charles Albert Gobat.

En el premio de 1903, los valores se repiten en William Randal CREMER, aunque sus orígenes son humildes y su progresión fue consecuencia de su esfuerzo personal, lo cierto es que al igual que los anteriores puede definirse a la altura de las décadas previas a la concesión como un político ya moderado, partidario del arbitraje y que ha trabajado intensamente en esta dirección hasta ver concluido en octubre de 1903, un Tratado anglo-francés que obligaba a los dos gobierno a arbitrar sus diferencias ${ }^{26}$ y cofundador con Passy de la Unión Inter-parlamentaria. Contará entre sus nominadores con Hans J. Horst y el grupo Inter-parlamentario sueco, lo que pone de manifiesto por una parte la fuerza que tienen como nominadores y por otra el círculo de nominaciones dentro de la Unión Inter-parlamentaria.

Cuando en enero de 1905, Cremer acuda a Cristianía, el presidente del Comité Jorgen Gunnarsson Løvland, dará un breve discurso de bienvenida donde de forma nítida quedará claro el objetivo del Comité en la dirección del derecho internacional, y se congratula; su mejor deseo será ver como «las ideas de la paz y el arbitraje florecen y ganan fuerza e influencia ${ }^{27}$.

De esta forma en los tres primeros años han sido premiadas las tres figuras visibles más relevantes de la Unión Inter-parlamentaria, los dos co-fundadores, Cremer y Passy y su Primer Secretario

${ }^{24}$ MLA style. «The Nobel Peace Prize 1902». Nobelprize.org. Nobel Media AB 2014. Web. 25 Aug 2015. http://www.nobelprize.org/nobel_prizes/peace/laureates/1902/ toast-speech.html.

${ }^{25}$ El criterio de premiar a un pacifista parlamentario y otro popular se repetirá en otros casos como con Renault y Moneta o Asser y Fried.

${ }^{26}$ Biographical Dictionary of Modern Peace Leader..ob. cit.. 182.

${ }^{27}$ MLA style. «The Nobel Peace Prize 1903». Nobelprize.org. Nobel Media AB 2014. Web. 21 Aug 2015. http://www.nobelprize.org/nobel_prizes/peace/laureates/1903/ welcome-speech.html. 
General, Gobat, y de la Oficina Internacional de la Paz, al incluir a Ducommun.

El círculo se cierra en 1904 con otro premio en la misma dirección al Instituto de Derecho Internacional (IDI). En el premio Libæk descubre la influencia del noruego Gregers Gram (1846.1929), que había sido representante del gobierno en Estocolmo y desde 1899 Juez de la Corte Permanente de Arbitraje de la Haya, y en 1904 miembro de IDI $^{28}$. Pero a mayor abundamiento ese año el puesto de Johannes Steen en el Comité, había sido ocupado por Nikolaus Matias Gjelsvik. Así pues nada de extrañar este premio. El IDI destaca entre sus objetivos, favorecer el progreso del derecho internacional y dentro de sus límites actuar en el «mantenimiento de la paz y la observancia de las leyes de la guerra ${ }^{29}$. No se puede dejar pasar por alto que contó con la nominación del presidente del Comité y Jørgen G. Løvland, y Georg G. Hagerup, entonces miembro del Instituto y que después también del Comité.

Cuando en agosto de 1912, en Noruega se dé la bienvenida a una representación del IDI, con motivo de una invitación del Gobierno, con motivo de celebrarse ese año la reunión anual de Instituto en Cristianía, la recepción correrá a cargo de Johannes Irgens, Ministro de Asuntos Exteriores, que presidirá la sesión de apertura. En su discurso $^{30}$ se pone de manifiesto nuevamente la sintonía del Gobierno con el premio, y se realza la importancia que se da al derecho como medio de resolución de conflictos, la idea de justicia entre los pueblos se considera una contribución vital para la paz. El acto también contó con la presencia del propio rey, y el cuerpo diplomático aparte de los miembros del Instituto, todo ello incide en el hecho de cómo el gobierno hace uso ya desde el principio del prestigio o fama que da el premio para tejer sus relaciones internacionales y así reconoce como por este medio Noruega, «se familiarizó más con el Instituto».

En definitiva de esta primera fase del premio cabe concluir la uniformidad del Premio, el Comité apoya sin fisuras la postura de las organizaciones pacifistas con las que se identifica. Ha sentado unas bases sobre las que se apoyan todos los premiados, que insistimos llama la atención que en estos años no ha dado entrada a nuevas per-

${ }^{28}$ LIBÆK Ivar. The Nobel Peace Prize: Some aspects...

${ }^{29} \mathrm{http} / / /$ justitiaetpace.org/status.php.

${ }^{30}$ MLA style. «The Nobel Peace Prize 1904». Nobelprize.org. Nobel Media AB 2014. Web. 21 Aug 2015. http://www.nobelprize.org/nobel_prizes/peace/laureates/1904/ welcome-speech.html. 
sonalidades, todas estaban definidas ya en la primera nominación. La sintonía entre nominadores y Comité es altamente sospechosa.

El premio correspondiente a 1905, se desarrolla a caballo entre una Noruega dependiente e independiente, en febrero cuando se cierra el plazo para las nominaciones, nada ha cambiado con relación a los años anteriores, sin embargo cuando se haga público el premio, el país acababa de estrenar su independencia. En diciembre, se anuncia el premio a Bertha von Suttner, y aunque aparentemente todo es normalidad, no es posible sustraerse a la idea de la tensión a que hubieron de estar expuestos muchos dirigentes noruegos y entre ellos los miembros del Comité, del abismo de la guerra al reconocimiento de independencia ${ }^{31}$. Es posiblemente un premio que no hubieran querido ni el Gobierno ni el Parlamento, será el primer año en que otorgándose a un sólo galardonado, no ha recaído en un parlamentario, ni experto jurista, aunque ello no significa una ruptura, ni mucho menos, con el pacifismo que premia el Comité. Suttner, que ya fue nominada en 1901 y desde entonces todos los años, apoyará a la Unión Inter-parlamentaria además ostenta la condición de vicepresidente de la Oficina Internacional de la Paz, la continuidad pues en este aspecto es evidente. Según Ivar Libæk ${ }^{32}$, Bjørnson y Lund, apostaron por Suttner, ya en 1904, aunque ese año la ausencia del primero pudo limitar sus posibilidades, lo consiguieron al año siguiente. De hecho ello explicaría que la presentación corriera a cargo precisamente de Bjørnson el 18 de abril de 1906, y que cuando tome la palabra el presidente del Comité, al mismo tiempo Ministro de Asuntos Exteriores Jørgen Gunnarsson Løvland, aunque resalte su figura lo hará con moderación $^{33}$, quedará lejos del interés que se mostrará ante el premio de año siguiente. En todo caso el premio a Suttner, sin duda será uno de los menos discutidos en la historia del Nobel de la Paz, posiblemente uno de los pocos que hubiera deseado su fundador, y Bjørnson, de quien Nobel era un profundo admirador ${ }^{34}$, era consciente de ello. La concesión de este premio será prácticamente el primer acto oficial del nuevo rey de Noruega, Haakon VII, que había llegado al país el 25 de noviembre, jurado el 27 y acudió al Instituto Nobel al anuncio el 10 de diciembre ${ }^{35}$.

${ }^{31}$ Sobre el papel jugado durante el proceso de independencia de algunos miembros del Comité véase REINAR, Mathias Brynildsen, «Lille land - hva da?

${ }^{32}$ LIBÆK Ivar. The Nobel Peace Prize: Some aspects ..., ob. cit., pág. 20-21.

${ }^{33}$ MLA style. «The Nobel Peace Prize 1905». Nobelprize.org. Nobel Media AB 2014. Web. 25 Aug 2015. http://www.nobelprize.org/nobel_prizes/peace/laureates/1905/speeches.html.

${ }^{34}$ SOHLMAN, Ragnar y otros. Los premios Nobel..., ob. cit. pág 782.

${ }^{35}$ REINAR, Mathias Brynildsen. «Lille land - hva da? 
Desde que el 7 de junio de 1905, el Parlamento Noruego destrona al rey Oscar de Suecia ${ }^{36}$. Hasta octubre la relación entre ambos países se tensa y sitúa próxima al enfrentamiento armado, los movimientos diplomáticos se intensifican con y entre las principales potencias europeas. Cuando la guerra parecía que era la única solución, el apoyo de las grandes potencias a la posición Noruega facilita que ambos países lleguen a un acuerdo y por la convención de $\mathrm{Karlstad}^{37}$, se resolvió pacíficamente el conflicto con el reconocimiento por parte de Suecia, de Noruega como país independiente.

En el periodo que media entre la independencia y la I Guerra Mundial, Noruega instauró el sufragio universal, en 1907 reconoció el derecho a voto de la mujer y en 1911 su acceso a todos los puestos de trabajo excepto a las carteras ministeriales, cosa que sucederá en 1916. Por otro lado en 1906, superados los momentos difíciles se romperá la unidad que los partidos habían mantenido en defensa de la independencia, lo que no impedirá que el Gobierno surgido en 1908, se muestre favorable a emprender reformas sociales, ni tampoco afectará a la composición del Comité. Noruega tratará de mantener relaciones privilegiadas con el mayor número de países, y se van a plantear nuevos retos y dificultades. Necesitará determinar sus límites territoriales, no sólo terrestres sino sobre todo el alcance de las aguas jurisdiccionales, cuestión sumamente relevante en un país con fuertes intereses marítimos, de forma que se impondrá la necesidad de suscribir pactos y tratados con otros países, especialmente los limítrofes. Esta nueva realidad, va a tener una relativa repercusión en el Premio Nobel, así junto a algún caso en que la motivación puramente política se manifieste de forma nítida en los demás la continuidad será indudable, de forma que en líneas generales, visto desde el punto de vista del premio estos acontecimientos se reflejan sólo relativamente, la composición del Comité se mantuvo relativamente estable, Johannes Steen que falleció en 1906 será sustituido por Carl Berner, miembro también del partido laborista y si se quiere el único cambio que en teoría podía ser significativo será el del liberal Bjørnson, el de menor perfil político pero sin duda la personalidad más conocida internacionalmente, por el conservador Georg Francis Hagerup, con lo que a nivel del Comité se pierde fuerza en la rama del pacifismo popular en favor de la vertiente jurídica. Solamente el primero de estos

${ }^{36}$ KENT, Neil. Historia de Suecia, Ed. Akal, Barcelona, 2011.

${ }^{37}$ Nombre de la localidad sueca donde se desarrollaron las conversaciones entre Suecia y Noruega y que desembocaron en un acuerdo que ratificado por los parlamentos de ambos países reconocería la independencia de Noruega. 
cambios se habrá reflejado en el Comité cuando se otorgan los premios de 1905 y 1906.

Restablecida la «normalidad» en 1906, se concede el premio más polémico del periodo al presidente de Estados Unidos, Theodore Roosevelt. La justificación formal será su intervención en el tratado de paz que puso fin a la guerra ruso-japonesa de 1904-1905, aunque hay motivos para pensar que hubo también otras razones ${ }^{38}$.

El discurso de presentación vuelve otra vez al campo político de la mano de Gunnar Knudsen, y las expresiones no pueden ser más nítidas, se involucra a todo el aparato político en el premio, se ensalza y halaga su figura, dirigiéndose al embajador de Estados Unidos (el premiado no estaba presente y el premio fue aceptado en su nombre por el embajador) en términos tales como: «En nombre del Parlamento Noruego... presento ... el premio Nobel de la Paz... y añado la solicitud de que transmita al presidente el saludo del pueblo noruego y su gratitud por todo lo que tiene hecho en la causa de la paz» para continuar con «el deseo de que este hombre eminente y superdotado, pueda ser bendecido... ${ }^{39}{ }$ \% En definitiva el premio no lo presenta un miembro del Comité, sino un representante político, que por otra parte sumaba a tal cargo su condición de armador con los consiguientes intereses en el transporte marítimo.

La naturaleza política de este premio tiene más argumentos, ni el número ni la calidad de los nominadores indican un apoyo masivo ${ }^{40}$, por su parte tampoco el informe emitido por Halvdan Koht era favorable y ponía de manifiesto no pocos aspectos discutibles de su política, asimismo sus actividades públicas e intervenciones de contenido bélico, previas a su acceso a la presidencia no lo hacían acreedor del premio. Ahora bien si hay que decir algo a su favor, y es que por primera vez, se justifica en un acontecimiento reciente acaecido, como exige el testamento dentro del «año transcurrido», cual es el tratado de paz en la guerra ruso-japonesa concluido en 1905.

Tras estos dos premios, de tan distinto significado, los entregados en los años siguientes, tienen el mismo común denominador que de los primeros, el Comité vuelve hacia los criterios previos a la declara-

${ }^{38}$ MLA style. «The Nobel Peace Prize 1905». Nobelprize.org. Nobel Media AB 2014. Web. 20 Aug 2015. http://www.nobelprize.org/nobel_prizes/peace/laureates/1905/speeches.html.

${ }^{39}$ MLA style. «The Nobel Peace Prize 1906 - Presentation Speech». Nobelprize.org. Nobel Media AB 2014. Web. 25 Aug 2015. http://www.nobelprize.org/nobel_prizes/ peace/laureates/1906/press.html.

${ }^{40}$ MLA style. «Nomination Database». Nobelprize.org. Nobel Media AB 2014. Web. 25 Aug 2015. http://www.nobelprize.org/nomination/archive/list.php.

(C) UNED. Revista de Derecho UNED, núm. 17, 2015 
ción de independencia, con algún matiz, así en muchos de ellos es fácil intuir la búsqueda de un equilibrio, ahora quizá más sutil, pero en todo caso perceptible entre los premiados, su actividad, y los países que indirectamente se consideran beneficiados, sin olvidar el cambio que supone la sustitución del escritor Bjørnson, por el casi veinte años más joven jurista Hagerup.

De esta forma por un lado el derecho internacional se convierte de nuevo en el protagonista de los premios, cuando se concede el de 1907, a Louis Renault, se busca de nuevo un experto jurista en derecho internacional, que ha desempeñado funciones políticas al más alto nivel y amigo personal por cierto de Francis Hagerup ${ }^{41}$. Además los críticos en Noruega entendieron que el premio se usó para reforzar la política exterior, y recibirá en Noruega una cálida recepción por parte de las autoridades de país ${ }^{42}$. El otro co-premiado Teodoro Moneta, es más un pacifista de acción, de la rama popular, fundador de la Unión Lombarda, director de la revista «La Vita Internationale» y que en 1906 había presidido el Congreso Internacional por la Paz celebrado en Milán. Como los primeros premios guarda un equilibrio entre un pacifismo jurídico o técnico encarnado en Renault y otro con una figura más popular que representa Moneta, y en el plano político también es posible encontrar un equilibrio de contenido político; en una Europa que en ese momento se encuentra profundamente dividida entre dos bloques, que parecen dirigirse irremisiblemente a la confrontación, la Triple Entente y la Triple Alianza ${ }^{43}$, es un premio a un nacional de un país de cada bloque, el equilibrio es necesario, a estas alturas la tensión es grande y el Comité se muestra sumamente sensible.

Otro premio, en el que también puede intuirse un equilibrio en este caso dirigido a los países nórdicos, es el de 1908. La guerra parece inminente, en el sur de Europa, se suceden acontecimientos preocupantes, se inicia una revuelta en el imperio otomano, Austria se anexiona Bosnia Herzegovina con la oposición de Rusia que inicia preparativos militares, a ellos se suma un regicidio en Portugal $^{44} \mathrm{y}$ en la guerra declarada en Persia Gran Bretaña apoyará al Sha. Todo ello parece justificar el alejamiento el premio de ese escenario y dirigirlo a los países nórdicos en la línea de reforzar la colaboración entre ellos,

${ }^{41}$ LIB/EK Ivar. The Nobel Peace Prize: Some aspects ..., pág. 25

42 Ídem ..., págs. $25-26$

${ }^{43}$ La Triple Entente agrupaba a Rusia, Francia y Reino Unido y la Triple Alianza englobaba a Alemania, Austría-Hungría e Italia.

${ }_{44}$ El 1 de febrero de 1908, son abatidos el rey Carlos I y el príncipe heredero Luis Felipe en el centro de Lisboa. 
concediéndoselo a Arnoldson y Bajer, si bien la condición de los premiados se mantendrá sin cambios; parlamentarios, partidarios del derecho y el arbitraje como forma de resolución de los conflictos. Klas Pontus Arnoldson, firme defensor de esta línea desde hace años y en la que insistió con motivo de la disolución de la unión sueco-noruega en 1905, con lo que el premio no deja de ser una recompensa por su posición en aquellos momentos, además fundador de la Liga sueca para la Paz y el Arbitraje ${ }^{45}$. La otra figura es un histórico del pacifismo el danés Fredrik Bajer, antiguo oficial de ejército, al que se atribuye la creación de la Oficina Internacional Permanente de la Paz, miembro del Consejo Inter-parlamentario. Como se ha dicho el premio puede ser considerado como un apoyo a la voluntad de acercamiento entre los países escandinavos ${ }^{46}$. Finlandia se encontraba sometida a Rusia, por lo que con el premio a un sueco y a un danés, al margen de la propia Noruega, abarcaba la totalidad de Escandinavia.

De nuevo al año siguiente un premio compartido, entre Beernaert y D’ Estournelles. Auguste Marie Francois Beernaert, de nuevo un estadista católico, belga, que promueve la aplicación del derecho entre las naciones, basado en la «moralidad católica y la tradición ${ }^{47}$ » jurista, diputado, ministro y jefe del Gobierno, miembro del Consejo Inter-parlamentario ${ }^{48}$ y representante de su país en las dos Conferencias de Paz de la Haya, presidente de la Asociación Internacional de Derecho y figura relevante de la Unión Inter-parlamentaria ${ }^{49}$. Paul D’Estournelles, francés, diputado, senador, miembro de la delegación francesa en las Conferencias de la Haya de 1899 y 1907, fundador de un grupo parlamentario en pro del arbitraje internacional, y promotor del tratado de arbitraje franco británico de 1903.

Creada por incitativa de Bajer premiado dos años antes, la Oficina Internacional de la Paz, obtiene el premio Nobel en 1910. La concentración de premios es total, el Comité trata de cerrar el círculo, al igual que hizo en 1904 con el premio al IDI. Sin negar el interés de la Organización y su papel en la dinámica pacifista del momento, la reiteración resulta cuanto menos discutible.

${ }^{45}$ MLA style. «Klas Pontus Arnoldson - Facts». Nobelprize.org. Nobel Media AB 2014. Web. 23 Aug 2015. http://www.nobelprize.org/nobel_prizes/peace/laureates/1908/arnoldson-facts.html.

${ }^{46}$ FOL, Jean-Jacques. Los Países Nórdicos en los siglos XIX y XX, Ed. Labor Barcelona, 1984, pág 116.

${ }^{47}$ Biographical Dictionary of Modern Peace Leader..., op. cit.,. pág. 69.

${ }^{48}$ Ídem. pág. 68

${ }^{49}$ MADRILEJOS, Mateo. En PRADO, J. Manuel, Los premio Nobel, op. cit., pág. 129. 
El último premio compartido de 1911, que reproduce por enésima vez los criterios anteriores, se otorgara de forma compartida entre Tobias Michael Carel Asser y Alfred Herman Fried. El primero, holandés, prestigioso jurista, miembro del Consejo de Estado, uno de los fundadores del IDI, representante de los Países Bajos en las Conferencias de Paz de la Haya, Juez del Tribunal de Arbitraje de la Haya, actuó como árbitro en el conflicto entre Estados Unidos y Rusia sobre el estrecho de Behring y con Méjico a propósito de los fondos piadosos de California ${ }^{50}$. En cuanto a Alfred Hermann Fried, periodista, fundador de la Sociedad de la Paz alemana ${ }^{51}$, desarrolló una sólida teoría sobre el pacifismo, que dio lugar a la creación por abogados internacionales en 1911 de la Asociación para el Entendimiento Internacional. Así pues un nuevo premio para el pacifismo jurídico y el popular.

Posiblemente la guerra de los Balcanes, cada vez más extendida, la intervención italiana en Tripolitania y Cirenaica, llevan al comité a no conceder premio el año 1912. Se aprovechará el de 1913, para conceder además de el de ese año a Henri La Fontaine, el de 1912 a Elihu Root, con independencia de la politización que pueda verse en este segundo, o que se aproveche aquel, mejor admitido para conceder este, desde el punto de vista que aquí nos interesa, vuelve el Comité a incidir en un premio al derecho y al arbitraje, sin perjuicio de incidir en la mejora de las relaciones con Estados Unidos.

Llama la atención el hecho de que el discurso de presentación de Root, no lo efectuó el presidente como lo venía haciendo de forma ininterrumpida desde 1907, sino el Secretario Ragnvald Moe, que no dudará en hacer lecturas positivas de la actividad de Root aunque sea desde puntos de vista discutibles como la intervención de Estados Unidos en Cuba o Filipinas, o el problema con los inmigrantes japoneses. Se mencionará su condición como presidente de la Fundación Carnegie, que tan estrechas relaciones e influencia económica tuvo en la Unión Inter-parlamentaria ${ }^{52}$.

En la misma línea el abogado y profesor de derecho internacional Henri La Fontaine, político, defensor del arbitraje del derecho internacional así como de la creación de un tribunal mundial e incluso que se dispusiera de la fuerza para hacer cumplir sus resoluciones y

${ }^{50}$ Se trata de la primera controversia internacional sometida al Tribunal de la Haya, y versaba sobre la compensación que tenía que pagar el gobierno de Méjico a los obispos católicos de California por la venta de los bienes correspondientes a las donaciones efectuadas para las misiones en esos territorios.

${ }^{51}$ Ídem., págs. 161-162.

52 Ver GROSSI, Verdiana. L'argent au service de la paix, en Pacifisme Europeen 1889-1914 Bruylant, Bruxelles, 1994, págs. 120-139. 
que al igual que muchos de los premiados anteriores participó en los congresos de paz, fue presidente de la Oficina Internacional de la Paz y figura destacada de la Unión Inter-parlamentaria ${ }^{53}$,

La relación de los premiados con la Unión Inter-parlamentaria y la Oficina Internacional de la Paz y entre estas es general, como se puede apreciar, al margen de los premios a Roosevelt y Root, son pocos los años en que no hayan resultado premiadas figuras relevantes de ambas

\begin{tabular}{c|c|c}
\hline AÑO & \multicolumn{1}{|c|}{ UNIÓN } & \multicolumn{1}{c}{$\begin{array}{c}\text { OFICINA INTERNACIONAL } \\
\text { DE LA PAZ }\end{array}$} \\
\hline 1901 & Frederick Passy (Cofundador) & $\begin{array}{l}\text { Frederick Passy (Miembro } \\
\text { del Consejo) } \\
\text { Elie Ducommun (Secretario } \\
\text { General) } \\
\text { Albert Gobat (Secretario } \\
\text { General) }\end{array}$ \\
1902 & Albert Gobat (Secr. General) \\
1905 & $\begin{array}{l}\text { William R. Cremer } \\
\text { (Cofundador) }\end{array}$ & $\begin{array}{l}\text { Bertha Von Suttner } \\
\text { (Vicepresidente) } \\
\text { Ernesto Moneta (Miembro } \\
\text { del Consejo) }\end{array}$ \\
1907 & $\begin{array}{l}\text { Frederick Bajer } \\
\text { August Beernaert }\end{array}$ & $\begin{array}{l}\text { Oficina Internacional de la } \\
\text { Paz } \\
1910\end{array}$ \\
1911 & $\begin{array}{l}\text { Alfred Fried (Miembro del } \\
\text { Consejo) } \\
\text { Henri La Fontaine (Miembro } \\
\text { del Consejo) }\end{array}$ \\
\hline 1913 & Henri La Fontaine & \\
\hline
\end{tabular}

Dominado en su mayor parte por el parlamentarismo y con el derecho internacional y el arbitraje como soportes ideológicos, no tendrá capacidad el Comité en estos años para dar entrada a otras figuras o movimientos que, con igual o mayor razón pueden considerase defensores de la paz, y que aún han asumido sacrificios personales en defensa de una idea de la paz, como pueden ser en el plano individual

${ }^{53}$ Biographical Dictionary of Modern Peace Leader.., ob. cit., pág. 538-9. 
el caso de Tolstoi, o el colectivo de la Oficina Socialista Internacional, por citar dos casos, de figuras que habían sido nominados.

Conocida la línea que han seguido los premios es evidente que Tolstoi nunca estuvo en la mente del Comité, planteamientos como «una aversión profunda hacia el liberalismo» negar que «el parlamentarismo o la secularización fuesen factores de mejora y progreso social ${ }^{54}$, su postura contra la propiedad privada, o la afirmación de que iniciativas como las Conferencias de Paz de la Haya, (tan caras al Comité Nobel) estaban «destinadas al más absoluto de los fracasos» ${ }^{55}$, no tenían cabida en un colectivo burgués, por más que en no pocos aspectos su proceder encaje mejor que muchos de los premiados en las disposiciones de Nobel, así cuando muestra repulsa a la guerra ${ }^{56}$, propone «cambiar: la violencia y el asesinato por la no participación en la violencia y los asesinatos ${ }^{57}$; o manifiesta que la casta militar «no sirve para nada ${ }^{58}$. Sólo recibirá una nominación para cada uno de los años 1901,1902 y $1909^{59}$.

En cuanto a la Oficina Socialista Internacional, correrá el mismo camino, aunque será nominada para 1912,1913 y 1914, el movimiento socialista representa, a pesar de su heterogénea composición un bloque pacifista muy activo en los años previos a la guerra. Los planteamientos moderados del Comité se encuentran igualmente muy distantes de las ideas socialistas, y aún más alejados de la radicalidad de algunas de sus figuras; la crítica de August Bebel, a países que como Estados Unidos o Francia, empleaban el poder del Estado para hacer fracasar huelgas o disparar contra los huelguistas en el Congreso de Amsterdam de 1904, o propuestas de otros líderes que defienden el recurso a la huelga en caso de guerra, que se planteó reiteradamente, aunque nunca con convicción, en los congresos Stuttgart 1907 o Copenhague $1910^{60}$ o posturas más radicales como el antimilitarismo de Gustave Hervé, no pueden ser admitidas por un gobierno liberal burgués.

${ }^{54}$ GORDILLO, Luis. En Pensamiento pacifista, PRAT, Enric (ed.), Ed, Icaria-Antrazyt, Barcelona, 2004, pág. 36.

${ }^{55}$ PRAT, Enric (ed). Pensamiento pacifista, PRAT, Enric (ed.), Ed, Icaria-Antrazyt, Barcelona, 2004, pág. 44.

${ }^{56}$ TOLSTÓI, Lev. Diarios (1895-1910), Ed. y trad. de Selma Ancira, Ed. Acantilado, Barcelona, 2003, pág. 134.

${ }^{57}$ TOLSTÓI, Lev. Diarios (1895-1910), Ed. y trad. de Selma Ancira, Ed. Acantilado, Barcelona, 2003, pág. 432.

${ }^{58}$ TOLSTÓI, Lev. Diarios (1895-1910), Ed. y trad. de Selma Ancira, Ed. Acantilado, Barcelona, 2003, pág. 23.

${ }^{59}$ MLA style. «Nomination Database». Nobelprize.org. Nobel Media AB 2014. Web. 24 Aug 2015. http://www.nobelprize.org/nomination/archive/list.php.

${ }^{60}$ COLE, G. D. A. Historia del pensamiento socialista, vol. III, La Segunda Internacional, págs. 57-97. 
Otros, ni siquiera serán propuestos a pesar de su clara postura en defensa de la paz, oponiéndose a la guerra incluso en la misma Alemania, como es el caso, con independencia de su evolución posterior de Karl Liebknecht, que en su condición de miembro del Reichstag, donde representa la extrema izquierda del SPD, votará en 1914 contra los créditos militares, fundará la Liga Espartaquista (Spartakusbund) en 1916 y será arrestado por gritar «abajo la guerra» en la Potsdamer Platz en pleno centro de Berlín ${ }^{61}$. Lo mismo puede decirse de Gustave Herve o Jean Jaures, y ello a pesar de que contará con la simpatía de Løvland ${ }^{62}$. Es claro que el bloque de nominadores tenía una visión relativamente próxima a la del Comité, y en ningún momento se planteaban la nominación de quienes surgían como sus enemigos políticos o defendían posturas contrarias a sus concepciones socioeconómicas. En definitiva el propio Schou ${ }^{63}$ al referirse al Comité a pesar de afirmar su independencia, reconoce que responde más bien a un "punto de vista liberalburgués» y «que sólo en años recientes han estado representadas directamente las opiniones socialistas» considerando el primer premio en esta dirección el concedido a La Fontaine en 1913.

Sea un movimiento pacifista u otro la guerra significará el fin de ambos, cuando los pacifistas burgueses se reúnan a finales de julio de 1914 tendrán que admitir la «futilidad de sus acciones en favor de la paz que había sido su razón de ser» ${ }^{64}$, y cuando los socialistas lo intenten, prevalecerá el nacionalismo y los esfuerzos por detener la guerra le costarán la vida al propio Jaurès ${ }^{65}$.

\section{CONCLUSIONES}

Frente a lo que pueda imaginarse el Premio Nobel de la Paz, en sus primeros años, responde a un esquema en gran medida planificado. Los premios lejos de ser el resultado de un complejo proceso de selección a fin de determinar en quien concurrían los méritos establecidos por Nobel para su concesión, son resultado de un sistema que se inicia antes de la concesión de los premios. En efecto en primer lugar, los miembros del Comité son en esencia representación del Par-

${ }^{61}$ MILZA, Pierre. De Versailles à Berlin 1919-1945. 3. a édition mise à jour, París, 1976, pág. 8.

${ }^{62}$ REINAR, Mathias Brynildsen. «Lille land - hva da?, ob. cit., pág. 74.

${ }^{63}$ SCHOU, August en SOHLMAN Ragnar y otros. Los premios Nobel y su fundador, Editorial Aguilar, Madrid, 1959, pág. 784.

${ }^{64}$ GROSSI, Verdiana. Le Pacifisme..., ob. cit. pág. 401.

${ }^{65}$ Asesinado el 31 de julio de 1914, cuando aún tenía esperanzas en detener la guerra. Su asesino fue liberado en 1919 tras 56 meses en prisión. 
lamento y la universidad, en los estatutos, se reconoce la posibilidad de que los premios sean otorgados no sólo a personas físicas sino a asociaciones, además se limita la capacidad de nominar a determinados colectivos, que paradójicamente reflejan el Parlamento y el Comité, al centrarse principalmente en miembros de los parlamentos nacionales, tribunales internacionales y determinados profesores universitarios.

Sobre estas bases los pacifistas nominados, van a ser fiel reflejo de los nominadores, parlamentarios y profesores universitarios especialmente juristas, en no pocos casos reuniendo ambas condiciones, y a partir de aquí la mayoría de los premiados responden a estos perfiles, y lógicamente en gran medida muy conservadores, como dice Verdiana Grossi este tipo de pacifista burgués pretendía ser a la vez «el precursor de un nuevo orden internacional y el heredero de una tradición humanista» ${ }^{66}$, o se hablará de un "pacifismo democrático» ${ }^{67}$, será un pacifismo tan teórico, que cuando se desate la guerra se convertirá en nacionalismo, o cuanto menos quedará en cuarentena. Al otorgar los premios a personas o asociaciones nominados año tras año, se incumple la condición de premiar un hacer durante «el año transcurrido" o cuanto menos de hechos que fueran recientes, asimismo muchos de los premiados, (entre las pocas excepciones podemos señalar la de Suttner, ya viuda, o Fried, al que las dificultades acompañaron toda su vida), distan de tener necesidades económicas.

Por último, y quizá lo más importante, es que desde el principio la finalidad que Nobel pretendía premiar, esto es «la fraternidad de las naciones y la abolición o reducción de los ejércitos permanentes así como la celebración y promoción de Congresos por la Paz», resulta claramente incumplida cuando se premia a especialistas o partidarios del derecho internacional o el arbitraje con la profusión que se hace en estos años. Es razonable pensar que si Nobel hubiera querido limitar el premio a los partidarios del arbitraje lo hubiera hecho, y fuera de ello no cabe equiparar unos méritos con otros. Aquellos responden a planteamientos idealistas, estos son sumamente prácticos y necesarios para un país escasamente poblado como Noruega que buscaba su propia seguridad, y que ninguna posibilidad tenía frente a las grandes potencias de la época, sin embargo el derecho internacional y el arbitraje le permitían defender sus derechos en un plano de igualdad, pero ello, no puede llevar a la conclusión de que la voluntad de Nobel se ha cumplido.

${ }^{66}$ GROSSI, Verdiana. Le Pacifisme..., ob. cit. pág. 13.

${ }^{67}$ PRAT, Enric (ed). Pensamiento pacifista, Ed, Icaria-Antrazyt, Barcelona, 2004, pág. 44 . 